\title{
Manual Anesthesia Record Keeping at a Tertiary Care Center: A Descriptive Cross-sectional Study
}

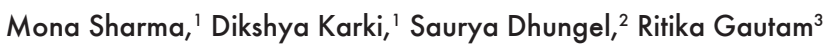 \\ 'Department of Anesthesiology, Kathmandu Medical College Teaching Hospital, Sinamangal, Kathmandu, Nepal, \\ 2Department of Epidemiology, School of Public Health, University of Washington, Seattle, United States of America, \\ ${ }^{3}$ KIST Medical College And Teaching Hospital, Imadol, Lalitpur, Nepal.
}

\begin{abstract}
Introduction: Intraoperative record form is one of the cardinal parts of anesthesia practices. Ideally, it should contain complete information about patients under anesthesia and intraoperative events. It serves as valuable information for subsequent patient management, research, or during medicolegal conditions. The objective of this study was to assess the practice and completeness of manual intraoperative anesthesia record keeping.
\end{abstract}

Methods: A descriptive cross-sectional study was conducted from May 1 to July 31, 2021, in the postoperative ward of Kathmandu Medical College, which is a multispecialty tertiary care center. Approval from the ethical committee of Kathmandu Medical College Teaching Hospital was obtained (Reference: 2603202105) before conducting the study. Convenience sampling was used. The data were entered in Microsoft Excel and statistical analysis was done using Statistical Package for the Social Sciences version 20. Point estimate was done at 95\% Confidence Interval and data present in numbers and percentages. We devised forty-two variables, which included demographics, personal identifiers, intraoperative events, anesthesia and airway management, intraoperative parameters, monitoring and medication.

Results: The overall completion rate was 202 (52.59\%) (47.6-57.57 at 95\% Confidence Interval). Out of 42 variables, the completion rate of 14 variables was less than $50 \%$. Among those were important parameters such as known allergies 94 (24.4\%), Body mass index $50(13 \%)$, intraoperative saturation of oxygen $104(27 \%)$, intraoperative electrocardiogram recording $107(27.8 \%)$, total fluid volume administered $45(11.7 \%)$, patient status on transfer $84(21.8 \%)$ had poor completion rate.

Conclusions: Our intraoperative record form shows poor completion rate, which was similar to other studies. many important variables were missing and had incomplete data.

Keywords: anesthesia; completion rate; intraoperative record; medico-legal.

\section{INTRODUCTION}

The anesthesia record is a document that provides information about perioperative care. This provides data on preoperative assessment, anesthesia management, vital parameters, and intraoperative events. ${ }^{1}$

The need for accurate and complete documentation cannot be overemphasized owing to medico-legal aspects, audits, future research and also the quality of care. ${ }^{2}$ The record provides valuable information to other healthcare providers who will be involved in patient care subsequently. ${ }^{3}$ The intraoperative phase involves anesthesia delivery, monitoring, management of critical events, which can impact proper and complete documentation; especially when patients are unstable. Work stress, manual recording, and practitioner interest are some of the factors, which affect the quality, and practice of documentation. ${ }^{4}$ Most hospitals in Nepal use manual intraoperative anesthesia record keeping.

The objective of this study is to assess the practice

Correspondence: Dr. Mona Sharma, Department of Anesthesiology, Kathmandu Medical College Teaching Hospital, Sinamangal, Kathmandu, Nepal. Email: dr.sharmamona@ gmail.com, Phone: +977-9843580393. 
and completeness of manual intraoperative anesthesia record keeping.

\section{METHODS}

A descriptive cross-sectional study was conducted from May 1 to July 31, 2021, in the postoperative ward of Kathmandu Medical College, which is a multispecialty tertiary care center. Approval from the ethical committee of Kathmandu Medical College Teaching Hospital was obtained (Ref: 2603202105) before conducting the study. Participants were informed about the nature of the study and informed consent was taken before enrollment, the only exclusion criteria were patient refusal. Since none of our patients refused, all were included. Convenience sampling was used. All record forms filled for elective surgeries performed were included. Based on a previous study, ${ }^{5}$ where the completion rates of records were $50 \%$, the sample size for our study was calculated using the formula:

$\mathrm{n}=\mathrm{Z}^{2} \times(\mathrm{p} \times \mathrm{q}) / \mathrm{e}^{2}$

$=(1.96)^{2} \times 0.5 \times(1-0.5) /(0.05)^{2}$

$=384.16$

$=385$

Where,

$\mathrm{n}=$ required sample size

$p=$ proportion of completeness of anesthesia record form $(50 \%)$ according to the previous study ${ }^{5}$

$q=1-p$

$\mathrm{e}=$ margin of error, $2 \%$

$Z=1.96$ at $95 \%$ Confidence Interval

The minimal sample size of 385 was calculated. A checklist was created based on our institution record form and policy statement from the Australian and New Zealand College of Anesthetists. ${ }^{6}$ The checklist was finalized after an initial pilot test. The checklist contained questions with three options "yes" for full data entry "incomplete" for partial data entry and "no" for missing data. The data was considered incomplete if full information was missing. For example in the case of date; year, month, and the day had to be written, for data requiring units, for example, weight in kilogram, age in months or year had to be mentioned.

A total of 42 variables were included which were demographics, personnel identifiers, intraoperative events, anesthesia and airway management, intraoperative parameters, monitoring and medication. A $100 \%$ completion rate was expected for all the variables. A Completion rate of less than $50 \%$ was regarded as a poor result and more than $90 \%$ completion was regarded as a good result. The data were entered in Microsoft Excel and statistical analysis was done using Statistical Package for the Social Sciences (SPSS) version 20. Narratives and tables were used to present data in numbers and percentages. Point estimate was done at $95 \%$ Confidence Interval and data present in numbers and percentages.

\section{RESULTS}

Out of a total of 385 record forms assessed in this study the overall completion rate was 202 (52.59\%) (47.6-57.57 at 95\% Confidence Interval). All the patients who had undergone surgery under general and regional anesthesia were included. Among all the record forms studied none had full information filled. The only record that was fully documented was the name of the anesthesia technique i.e. $100 \%$. The records were legible $355(92.2 \%)$ times. The overall completion rate of the chart was only $202(52.59 \%)$.

The patients' name was recorded in full in 377 (98\%) charts. It was the most frequently recorded indicator. This was followed by gender $314(81.6 \%)$. The age of the patient was incomplete in 127 (33.0\%); these records had no mention of years or months. The inpatient number was filled only in 246 (63.9\%) in the record form. Among preoperative details, body mass index (BMI) was filled only in 50 (13\%) patients. The mention of known allergies was missing in 291 records (75.6\%) (Table 1).

\begin{tabular}{|llll|}
\hline $\begin{array}{l}\text { Table 1. Intraoperative anesthesia record form completion rate for demographic and other baseline patient } \\
\text { information }(\mathbf{n = 3 8 5 ) .}\end{array}$ & \multicolumn{1}{l}{} \\
\hline Indicators & $\begin{array}{l}\text { Documentation rate } \mathbf{n}(\%) \\
\text { Full data }\end{array}$ & Incorrect/Partial data & Missing data \\
Name & $377(97.9)$ & $8(2.1)$ & 0 \\
Age & $258(67)$ & $102(26.5)$ & $25(6.5)$ \\
Gender & $314(81.6)$ & $10(2.6)$ & $61(15.8)$ \\
Weight & $194(50.4)$ & $5(1.3)$ & $186(48.3)$ \\
Ward/ bed no & $187(48.5)$ & $0(0)$ & $198(51.4)$ \\
Baseline vitals & $203(52.7)$ & $177(46)$ & $5(1.3)$ \\
Operating theatre number & $229(59.5)$ & 0 & $156(40.5)$ \\
ASA* status & $205(53.2)$ & 0 & $180(46.8)$ \\
Known allergies & $94(24.4)$ & 0 & $291(75.6)$ \\
\hline
\end{tabular}


${ }^{*}$ ASA American Society of Anesthesiologists.

The name of the anesthetist was recorded with full documentation 371 (96.4\%), likewise, the name of the procedure was mentioned 362 (94\%). However, the total volume of fluids received, mention of critical events was missing in 228 (59.2\%), 260 (67.6\%), $234(60.8 \%)$ records respectively. During the study duration, 258 cases were done under regional anesthesia. The site of regional anesthesia was fully documented in 157 (60.8\%) and completely missing in the rest 101 (39.2\%). Among 127 patients receiving general anesthesia, full documentation of the type of airway device used was done in 77 (60.6\%) records, laryngoscopy grade was mentioned in full data in 118 (92\%) (Table 2).

\begin{tabular}{|llll|}
\hline \multicolumn{4}{|l}{ Table 2. Intraoperative anesthesia record form completion rate for intraoperative events and anesthesia technique. } \\
\hline Indicators & Full data & Documentation rate $\mathbf{n}(\%)$ & Missing data \\
Date of surgery & $275(71.4)$ & $5(1.3)$ & $105(27.3)$ \\
Name of surgeon & $252(66.5)$ & $128(33.2)$ & $5(1.3)$ \\
Name of anesthetist & $371(96.4)$ & $9(2.3)$ & $5(1.3)$ \\
Name of the procedure & $362(94)$ & $18(4.7)$ & $5(1.3)$ \\
Type \&size of airway device used & $77(60.6)$ & $47(37)$ & $3(2.4)$ \\
Laryngoscopy grade & $118(92.1)$ & 0 & $9(7.9)$ \\
Ventilator mode and parameters & $48(37.7)$ & $54(42.5)$ & $25(19.8)$ \\
Fresh gas flow & $53(41.7)$ & $23(18.1)$ & $51(40.2)$ \\
Open or closed circuit & $60(47.2)$ & $27(21.2)$ & $31.6(30.7)$ \\
Fluid type & $354(91.1)$ & $8(2.1)$ & $23(6)$ \\
Total fluid volume & $45(11.7)$ & $80(20.8)$ & $260(67.6)$ \\
Total time for surgery & $176(45.7)$ & $143(37.1)$ & $66(17.1)$ \\
Total time for anesthesia & $136(35.3)$ & $157(40.8)$ & $92(24)$ \\
Patient's status on transfer & $84(21.8)$ & $52(13.5)$ & $249(64.7)$ \\
Mention of intraoperative critical events & $119(30.9)$ & $32(8.3)$ & $234(60.8)$ \\
\hline
\end{tabular}

The drug dosage was mentioned with full documentation in $347(90.1 \%)$ of cases; however, the drug route was missing in all cases. Likewise, the time at which the drug was given was missing in 360 (93.5\%). An extremely important detail of intraoperative vitals like the saturation of oxygen $\left(\mathrm{SPO}_{2}\right)$ was missing in 212 $(55.1 \%)$ of records. Documentation of care of pressure points, blood loss was completely missing in 257 (66.8\%), 208 (54\%) respectively (Table 3).

\begin{tabular}{|llll|}
\hline \multicolumn{4}{|l|}{ Table 3. Intraoperative anesthesia record form completion rate for intraoperative monitoring and medications. } \\
\hline Indicators & \multicolumn{3}{c|}{ Documentation rate n (\%) } \\
& Full data & Incorrect/Partial data & Missing data \\
Patient position & $258(67)$ & 0 & $127(33)$ \\
IV/arterial/CVP line size & $288(74.8)$ & $33(8.6)$ & $64(16.6)$ \\
tIV/ arterial/CVP line cannulation site & $303(78.7)$ & $59(15.3)$ & $23(6)$ \\
Drug dosage & $347(90.1)$ & 0 & $38(9.9)$ \\
Drug route & 0 & 0 & $385(100)$ \\
Drug timing & $25(6.5)$ & 0 & $360(93.5)$ \\
Intraoperative HR & $237(61.6)$ & $132(34.3)$ & $16(4.2)$ \\
Intraoperative BP & $293(76.1)$ & $76(19.7)$ & $16(4.2)$ \\
Intraoperative SPO & $104(27)$ & $69(17.9)$ & $212(55.1)$ \\
Intraoperative rhythm (ECG) & $107(27.8)$ & $120(31.2)$ & $158(41)$ \\
Blood loss & $107(27.8)$ & $70(18.2)$ & $208(54)$ \\
Care of pressure points & $128(33.2)$ & 0 & $257(66.8)$ \\
Urine output & $129(33.5)$ & 0 & $256(66.5)$ \\
Monitors used & $174(45.2)$ & $148(38.4)$ & $63(16.4)$ \\
\hline
\end{tabular}

†IV intravenous, CVP central venous pressure

\section{DISCUSSION}

None of the charts were filled completely. The overall completion rate of the chart was only 202 (52.59\%). Sub par documentation of manual record system has been documented in many previously conducted

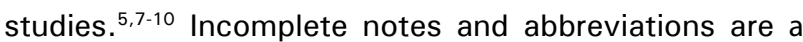
source of weak defense during litigations. Apart from that, information derived from record forms is of extreme value to subsequent practitioners who may give anesthesia services to the patient. ${ }^{2,3}$ 
Less than $50 \%$ completion rate was seen for many variables. Only $50.4 \%$ documentation of weight and missing data of BMl suggests that inaccurate dosage of drugs, ventilation parameters, airway equipment size and fluid management. This could result in poor quality of anesthesia management. Our finding correlates with other studies conducted. ${ }^{5}$

Demographic data like name, age and gender had more than $80 \%$ completion rate in this study. Some studies conducted in Africa have published results where these parameters had a poor completion rate, especially for the name of the patient. 5,7 This could be because of long names in the African continent. However, inpatient number and ward or bed number were fully recorded only in $63.9 \%$ and $48.5 \%$. These can lead to misidentification of patients with similar names. Allergic reactions in the intraoperative setting can be devastating especially in case of late diagnosis. Therefore, patients with known allergies have to be identified. However, in our findings, only $24.4 \%$ of records form had mentioned if the patient had any allergies. Catastrophic complications can occur when vital information as history of allergic reactions is missing in the record form.

Intraoperative vital parameters recorded in our study were heart rate, rhythm, oxygen saturation and blood pressure recorded every 5 minutes. The complete record of these parameters was poor especially for $\mathrm{SPO}_{2}$ and rhythm recorded by electrocardiograph (ECG), which was $27 \%$ and $27.8 \%$ respectively. This implies late detection and management of critical events, which in turn affects overall patient outcome. Such similar findings are depicted in other studies performed by Gebremedhn, et al. ${ }^{8}$ and Raff, et al. ${ }^{9}$

Drug dosing is of extreme importance in anesthesia, many factors play into action such as age, weight, comorbidities etc. In this study drug dose was fully mentioned in $90.1 \%$ of cases, however, the completion rate for drug timing was only $6.5 \%$ and the drug route was missing in all record forms. In previous studies done have shown a higher percentage of recording rates for these variables. This could be because of a structured intraoperative form, that we use, drug route and timing is not mentioned, nevertheless, it should have been recorded..$^{5,8}$

The completion rate of variables like ventilator parameters, fresh gas flow, total fluid given, urine output, check of pressure points, intraoperative critical events, status of patient on transfer were poor that is below $50 \%$. This poor documentation is sub-par of expected completion rate. This could signify poor quality of perioperative anesthesia services. These data could be against anesthesia service providers, as something that is not written did not happen. Therefore, where litigation claims are made, these under documented forms give us a lower hand.

However, variables such as name of the anesthetist, name of the procedure, laryngoscopy grade, fluid type and drug dosage was fully completed in more than $90 \%$ record forms. Similar high documentation rate has been recorded in studies conducted previously. ${ }^{8-10}$

Although many studies have included postoperative orders in anesthesia form, in our center we record postoperative order in another form, hence was not included in this study.

Our findings suggest that there are significant deficiencies in the adequate documentation of anesthetic record form. This has been shown mainly in recording of baseline and intraoperative vitals namely saturation of oxygen, blood loss, care for pressure points, mention of critical events, drug route and timing. These findings suggest that our intraoperative record form serves as a poor document for research, medico legal document and data for future reference. There are reports suggesting that critical events or complications that occur are intentionally not recorded for the fear of litigation, blame by colleagues and disciplinary action by the hospital management. ${ }^{11}$

A study conducted in 2015 has shown that when awareness is created about the importance of anesthesia record form by teaching and regular audit can improve the overall completion rate of the record form. ${ }^{12}$

According to new studies limitation of manual recording, can be over come by computerized record-keeping; as it has certain advantages like accurate record of intraoperative vital parameters, better quality of care and regulatory compliance. ${ }^{13,14}$

This study has limitation as it was performed at one teaching hospital and does not reflect the practices at other hospitals. Our study does not include preoperative chart, which contains pre-anesthetic evaluation of the patient. Nor does our study include postoperative order. Besides these, we have only included elective surgeries. Studies have shown that completion rate of intraoperative record form for emergency surgeries are poor. ${ }^{1,10}$

\section{CONCLUSIONS}

Our intraoperative record form shows a poor completion rate, many important variables were missing, and had incomplete data. Important parameters like the history of allergies, intraoperative saturation of oxygen and electrocardiogram recording, information about drugs route and intraoperative total fluids, patients' status on transport from operating theatre had less than $50 \%$ 
completion rate, i.e. poor result.

\section{ACKNOWLEDGMIENTS}

We would like to acknowledge all our patients and postoperative nursing staff for supporting the study.

\section{Conflict of Interest: None.}

\section{REFERENCES}

1. Elhalawani I, Jenkins S, Newman N. Perioperative anesthetic documentation: Adherence to current Australian guidelines. J Anaesthesiol Clin Pharmacol. 2013 Apr;29(2):211-5.

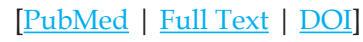

2. Mathioudakis A, Rousalova I, Gagnat AA, Saad N, Hardavella G. How to keep good clinical records. Breathe (Sheff). 2016 Dec;12(4):369-73. [PubMed | Full Text | DOI]

3. Parakh S C. Legal Aspect of Anaesthesia practice. Indian J Anaesth 2008; 52(3):247-57. [Full Text]

4. Marco AP, Buchman D, Lancz C. Influence of form structure on the anesthesia preoperative evaluation. J Clin Anesth 2003; 15(6):411-7. [ PubMed | DOI]

5. Zemedkun A, Mulugeta H, Getachew H, Destaw B, Mola S, Milkias M. Assessment of Manual Intraoperative Anesthesia Record-Keeping Practice at Dilla University Referral Hospital, Dilla, Ethiopia. Open Access Surgery. 2021;14:1-7. [DOI]

6. Australian and New Zealand College of Anaesthetists Professional Document PS06. Guideline on the anaesthesia record: recommendation; 2020. [Full Text]

7. Woldegerima Y, Kemal S. Clinical audit on the practice of documentation at preanesthetic evaluation in a specialized university hospital. Int J Surg Open. 2019;16:1-5. [ Full Text I DOI]

8. Gebremedhn EG, Nagaratnam V. Audit on anaesthetic record completeness at a university teaching hospital operation theater of low-resource setting. J Anesth Crit Care Open Access. 2017;8(2):2-6. [Full Text | DOI]

9. Raff M. An audit of anaesthetic record keeping. South Afr J Anaesth Analog. 2003;9(3):7-9. [DOI]

10. Ige FO, Adesina $\mathrm{K}$, Fatoba M. Completeness of manual anaesthesia records in a tertiary facility in Nigeria. J Med Trop. 2017;19(2):86-9. [Full Text]

11. Heard GC, Sanderson PM, Thomas RD. Barriers to adverse event and error reporting in anesthesia. Anesth Analg 2012;114(3):604-14. [PubMed | Full Text | DOI]

12. Olateju SO, Adenekan AT, Owojuyigbe AM. The effect of teaching on the completeness of the anesthesia record charts for obstetric subarachnoid blocks in a low resource area hospital. J Obstet Anaesth Crit Care 2015;5:16-21. [Full Text | DOI]

13. Palaniswamy SR, Jain V, Chakrabarti D, Bharadwaj S. Completeness of manual data recording in the anaesthesia information management system: A retrospective audit of 1000 neurosurgical cases. Indian J Anaesth. 2019;63(10):797-804. [uuMed | Full Text | DOI]

14. Shear TD, Deshur M, Lapin B, et al. Documentation and treatment of intraoperative hypotension: electronic anesthesia records versus paper anesthesia records. J Med Syst. 2017;86(41):1-8. [PubMed | Full Text | DOI] 